\title{
LEOPARDI, OS ANTIGOS E A TRADUÇÃO
}

\author{
Andréia Guerini* \\ Universidade Federal de Santa Catarina \\ Procad-UFMG
}

\begin{abstract}
RESU MO
Este artigo tem como objetivo analisar a relação do escritor italiano Giacomo Leopardi (1798-1837) com os antigos e de como a tradução de autores gregos e latinos foi importante para intensificar esta relação e contribuir para a formação deste que viria a ser um dos mais importantes escritores do século XIX italiano.
\end{abstract}

PALAVRAS - CHAVE

Leopardi, tradução, Literaturas clássicas

escritor italiano Giacomo Leopardi sempre esteve ligado ao mundo dos antigos. São vários os aspectos da sua obra que nos levam a esta constatação. Do epistolário (1807 a 1837) ao Zibaldone di Pensieri $(1817-1832)^{1}$ e da poesia aos textos em prosa não faltam referências ao mundo clássico. ${ }^{2}$ Afinal, como nos lembra o grande estudioso de Leopardi, Francesco De Sanctis, não "dobbiamo dimenticare che Leopardi s'è formato su'classici, e che nella sua opera si vede l'influsso di certi modelli e di certi fini preconcetti". ${ }^{3}$

Essa relação com o mundo clássico é descrita em uma carta de 05 de dezembro de 1817 endereçada ao grande amigo Pietro Giordani, em que Leopardi fala sobre o seu método de estudo e dos projetos:

(...) vo leggendo i miei Classici, Greci la mattina, Latini dopo pranzo, Italiani la sera; e così penso di durare un altro annetto, non iscrivendo fuori che qualche bagattella che ho in testa, e limandone due o tre altre già fatte, dopo il quale impratichitomi bene del greco e arricchitomi dell'oro dei Classici, fo conto di uscire in campo con una solenne traduzione (tanto solenne quanto posso darla io) e poi lasciar fare alla inclinazione e alla fortuna. ${ }^{4}$

\footnotetext{
*andreia.guerini@gmail.com

${ }^{1}$ Livro de 4.000 páginas manuscritas, em que Leopardi se revela um ensaísta de primeira grandeza, podendo ser comparado a Montaigne, Croce e Valéry.

${ }^{2}$ Nesse sentido, Alfredo Bosi observa: "De Leopardi os mais antigos documentos, entre cartas e páginas de erudição redigidas desde os doze anos de idade, revelam uma existência murada, toda entregue às imagens dos Antigos e alheia ao que não fosse a atenta refacção de uma vida remota, sobre cujos vestígios se debruçaria perdendo a saúde em sete anos de 'studio matto e disperatissimo." BOSI. A natureza, os antigos: Leopardi tradutor, p. 158.

${ }^{3}$ DE SANCTIS. Studio su Giacomo Leopardi, p. 262.

${ }^{4}$ LEOPARDI. Epistolario, p. 163.
} 
O lugar da tradução dos clássicos na vida literária de Leopardi já havia sido, de alguma maneira, ressaltado também pelo próprio autor quando, em carta de 21 de março de 1817 ao amigo Giordani, diz "Quando ho letto qualche classico, la mia mente tumultua e si confonde. Allora prendo a tradurre il meglio, e quelle bellezze per necessità esaminate e rimenate a una a una, piglian posto nella mia mente, e l'arricchiscono e mi lasciano in pace (...)." ${ }^{5}$

Assim, em um dos mais recentes livros que trata de aspectos da relação de Leopardi com os antigos, Il sogno di um'ombra: Leopardi e la verità delle illusioni, Lorenzo Polato, ao analisar as conceitos de "imaginação" e "ilusão" e a relação de Leopardi com os antigos, afirma: "Leopardi tiene lo sguardo rivolto al mondo antico: si apre allora uno scenario vastissimo in cui l'evocazione della sapienza antica s'intreccia con una dimensione dell'immaginare che diviene modernamente modo della soggettività, potenza creativa e autonoma." 6

Se tomarmos os elementos biobibliográficos de Leopardi, que teve uma vida "presto cominciata e presto finita", ${ }^{7}$ descritos por Antonio Ranieri, em Sette anni di sodalizio con Giacomo Leopardi, vários deles nos levam ao mundo clássico/antigo. O primeiro se relaciona à vasta biblioteca da casa paterna. Nela, segundo Ranieiri, Leopardi, que já sabia o italiano e latim, "imparò miracolosamente da se stesso, non solo la [lingua] francese, la spagnuola e l'inglese, ma ancora, quel ch'è assai piú, la greca e l'ebraica, nella quale giunse insino a disputare con alcuni dotti ebrei anconitani." ${ }^{8}$ O segundo elemento são as características que Ranieri apresenta em relação ao homem e ao intelectual Leopardi, retomadas mais tarde pela crítica em geral, ${ }^{9}$ ou seja, a de que o grande escritor italiano era um homem de grande talento, pois nele estavam unidos "due elementi quasi incompatibili, una gran fantasia e un gran raziocinio. La rarità della congiunzione di questi due elementi, e la frequenza della loro separazione, forma la rarità dei grandi ingegni, e la frequenza dei mediocri”. E continua:

(...) Leopardi, in cui quella congiunzione fu maravigliosa, conquistate nelle lingue le chiavi dello scibile ovvero dell'universo, studiò prima l'applicazione che vi fecero del loro ingegno i grandi uomini o antichi o moderni che lo avevano preceduto, e poi vi applicò il suo

\footnotetext{
${ }^{5}$ LEOPARDI. Epistolario, p. 71.

${ }^{6}$ POLATO. Il sogno di um'ombra: Leopardi e la verità delle illusioni, p. 15.

${ }^{7}$ DE SANCTIS. Studio su Giacomo Leopardi, p. 5.

${ }^{8}$ RANIERI. Notizia intorno alla vita e agli scritti di Giacomo Leopardi, p. 88. Esse texto também está disponível em: < http://www.classicitaliani.it/Ranieri/Ranieri_Notizia_Leopardi.htm>. Acesso em: 19 fev. 2009.

${ }^{9}$ Vale, contudo, lembrar que a partir da década de 1940 do século XX, novos estudos foram publicados como os de Walter Binni e Cesare Luporini, ambos de 1947 e, mais tarde, o de Sebastiano Timpanaro de 1965 que destacam os "pensamentos" e a figura cultural de Leopardi, mas ainda não estudam Leopardi no seu conjunto. Assim, concordo com Bollati quando ele diz que não se pode estudar Leopardi sem integrar todos esses aspectos/fases: filólogo, poeta, filósofo, ou o pensador e o homem militante junto com o escritor e o poeta. BOLLATI. Giacomo Leopardi e la letteratura italiana, p. 87. Leopardi também ganha um importante estudo, o de Antonio Prete, Il pensiero poetante, de 1980, que vai na direção sugerida por Bollati, pois promove um diálogo permanente entre o "pensiero poetante" e a "poesia pensante”. PRETE. Il pensiero poetante. Saggio su Leopardi, p. 49.
} 
proprio". O terceiro elemento que se agregou aos dois primeiros foi a "doença", "a dor", "la parte piú inesplicabile dell'inesplicabile mistero dell'universo. (...) Assim, o poeta de "L'Infinito" teve "il maggior bene, l'intelletto, commisto col maggior male, il dolore. ${ }^{10}$

A conjunção desses três elementos - fantasia/imaginação, raciocínio e dor - está ligada a três fases da evolução intelectual de Leopardi, isto é, a de ser, primeiro, um grande filólogo para depois se tornar um grande poeta (fantasia) e, por fim, para chegar ao filósofo, isto é, ao raciocínio: "Dunque il Leopardi fu prima gran filologo, poi gran poeta, poi gran filosofo. E per intendere la vera natura del suo ingegno, è mestieri di studiarlo ordinatamente sotto ciascuna delle tre grandi forme che assunse."11

Essa divisão proposta por Ranieri já estava no Zibaldone, pois em um fragmento de 19 de setembro de 1821, Leopardi se descreve por etapas: a primeira do filólogo, em seguida a do poeta e depois a do prosador ou filósofo:

Le circostanze mi avevan dato allo studio delle lingue, e della filologia antica. Ciò formava tutto il mio gusto: io disprezzava quindi la poesia. Certo non mancava d'immaginazione, ma non credetti d'esser poeta, se non dopo letti parecchi poeti greci. (Il mio passaggio però dall'erudizione al bello non fu subitaneo, ma gradato, cioè cominciando a notar negli antichi e negli studi miei qualche cosa più di prima ec. Così il passaggio dalla poesia alla prosa, dalle lettere alla filosofia. Sempre assuefazione.) Io non mancava nè d'entusiasmo, nè di fecondità, nè di forza d'animo, nè di passione; ma non credetti d'essere eloquente, se non dopo letto Cicerone. [1742] Dedito tutto e con sommo gusto alla bella letteratura, io disprezzava ed odiava la filosofia. I pensieri di cui il nostro tempo è così vago, mi annoiavano. Secondo i soliti pregiudizi, io credeva di esser nato per le lettere, l'immaginazione, il sentimento, e che mi fosse al tutto impossibile l'applicarmi alla facoltà tutta contraria a queste, cioè alla ragione, alla filosofia, alla matematica delle astrazioni, e il riuscirvi. Io non mancava della capacità di riflettere, di attendere, di paragonare, di ragionare, di combinare, della profondità ec. ma non credetti di esser filosofo se non dopo lette alcune opere di Mad. di Staël. ${ }^{12}$

No fragmento acima, Leopardi não apenas explicita as suas fases, mas também liga o seu percurso primeiro aos poetas gregos, para depois passar por Cícero e chegar aos modernos, através de Mme. de Stäel. No caso da influência grega, Leopardi não cita nenhum poeta em particular, fala no plural, o que nos leva a constatar que os poetas gregos, dos mais aos menos conhecidos, tiveram uma forte influência sobre o autor e contribuíram para que ele se tornasse um dos principais poetas do século XIX italiano, a ponto de Ugo Dotti afirmar que "La poesia dei classici antichi è comunque ancora, e lo sarà sempre, un modello per Leopardi". ${ }^{13}$

Antonio Prete observa em relação ao fragmento de 19 de setembro de 1821 que "Lesercizio della lettura [em Leopardi] produce la volontà della scrittura, rafforza la possibilità della scrittura, determina la scelta di uno stile, o di un gusto, o di una disciplina". ${ }^{14}$

\footnotetext{
${ }^{10}$ RANIERI. Notizia intorno alla vita e agli scritti di Giacomo Leopardi, p. 88.

${ }^{11}$ RANIERI. Notizia intorno alla vita e agli scritti di Giacomo Leopardi, p. 88-89.

${ }^{12}$ LEOPARDI. Zibaldone di Pensieri.

${ }^{13}$ DOTTI. Storia della letteratura italiana, p. 382.

${ }^{14}$ PRETE. Il pensiero poetante. Saggio su Leopardi, p. 159.
} 
Analogamente, poderíamos dizer que o mesmo acontece com Leopardi tradutor, pois se quisermos entender sua relação com os antigos, temos também de analisá-lo por este viés, porque essa atividade assume especial importância na sua formação como escritor, como veremos a seguir.

Foi na transição da infância para a adolescência que Leopardi escreveu as suas obras de maior erudição ${ }^{15}$ e deu início à sua atividade de tradutor, pois estava particularmente fascinado pelo mundo grego e latino, em geral, e pela literatura, em particular. Em uma carta a Giordani de 21 de março de 1817, Leopardi diz:

(...) quando ho letto qualche classico, la mia mente tumultua e si confonde. Allora, prendo a tradurre il meglio, e quelle bellezze per necessità esaminate e rimenate a una a una, piglian posto nella mia mente e l'arricchiscono e mi lasciano in pace. ${ }^{16}$

Nesse sentido, Lorenzo Polato afirma que com o mundo antigo Leopardi

aveva dialogato fin dalla fanciullezza e su di esso (o mundo antigo), divenuto filosofo, aveva altrettanto a lungo meditato. Ora é come se fossero giunte a maturazione insieme sia un'esperienza più che decennale di esercizio poetico, filologico, di traduttore, sia il parallelo lavoro del pensiero. ${ }^{17}$

Vale lembrar que ainda muito jovem Leopardi traduziu os idílios de $\mathrm{Mosco}^{18} \mathrm{e}$, mais tarde, a Batracomiomaquia, do pseudo-Homero, a Titanomaquia, de Hesíodo, o segundo livro de Eneida e o primeiro da Odisseia. As várias traduções que Leopardi realizou serviram de preparação para sua obra. ${ }^{19}$

Rolando Damiani, em All'apparir del vero - vita di Giacomo Leopardi, observa que Leopardi "ha scoperto traducendo il piacere gratuito della letteratura, l'amore per il bello coltivato privatamente". Ademais, afirma que é com as traduções que Leopardi se abre para a poesia, filólogo que era até então, porque

La traduzione degli Idilli di Mosco diverge dal progetto erudito-filologico (...). Essa nasce sotto il segno della predilezione letteraria, di un gusto individuale (...) Leopardi ha scoperto traducendo il piacere gratuito della letteratura, l'amore per il bello coltivato privatamente. (...) Nei poeti antichi, che legge e riversa nella propria lingua, Leopardi avverte qualcosa di suo (...). La catena dell'erudizione, i vincoli del tradurre, le dure leggi dell'emendatore di testo gli hanno insegnato la libertà della letteratura, il gusto di creare e di dirsi che ne costituiscono l'essenza. ${ }^{20}$

\footnotetext{
${ }^{15}$ São, por exemplo, desse período, Storia dell'astronomia (1813), Saggio sopra gli errori popolari degli antichi (1815).

${ }^{16}$ LEOPARDI. Epistolario, p. 69.

${ }^{17}$ POLATO. Il sogno di um'ombra: Leopardi e la verità delle illusioni, p. 30-31.

${ }^{18}$ As traduções dos Idílios de Mosco por Leopardi estão disponíveis no endereço: <http:// www.classicitaliani.it/leopardi/traduzioni/Leopardi_discorso\%20Mosco.htm>.

${ }^{19}$ Como bem lembra Alfredo Bosi, o poeta de "L'Infinito" não apenas traduziu os gregos e latinos, pois "o entusiasmo de Leopardi pela beleza dos textos antigos leva-o a compor diretamente em grego duas odes que finge serem de autor ignorado" e as traduz para o latim. BOSI. A natureza, os antigos: Leopardi tradutor, p. 166.
}

${ }^{20}$ DAMIANI. All'apparir del vero - vita di Giacomo Leopardi, p. 68. 
Para o grande historiador Francesco De Sanctis, na tradução dos idílios de Mosco, por exemplo, temos um primeiro indício da poética leopardiana, pois, provavelmente, foi nele que Leopardi se inspirou para compor seus idílios. Segundo o crítico italiano:

l'idillio leopardiano non ha niente di comune col significato che si dà generalmente a questa maniera di poesia. Non è descrizione della vita campestre, con dialoghi tra pastori, o pescatori, opera spesso di civiltà avanzata e stanca, che, mancato ogni degno scopo della vita, cerca nuovi stimoli negli ozii campestri. Forse per questo Leopardi più tardi cancellò quel nome d'idilli e diè a tutte le sue poesie un nome comune, Versi o Canti. ${ }^{21}$

Em 15 de novembro de 1816, em carta ao editor veneziano Antonio Fortunato Stella, Leopardi fala a respeito do ensaio sobre sua tradução dos poemas de Mosco, que ele afirma em carta a Cancellieri de 20 de dezembro de 1816 ter traduzido "Non un solo Idillio (...), ma l'intera traduzione delle sue poesie".22

A tradução ocupa um lugar de destaque na vida literária de Leopardi como já ressaltado também pelo próprio autor não só na carta de 21 de março de 1817 , citada acima, mas também em outras, sempre a Giordani, como a de 30 de abril de 1817, na qual ele aponta a necessidade de se traduzir bons autores pois:

(...) la proprietà de' concetti e delle espressioni sia appunto quella cosa che discerne lo scrittor Classico dal dozzinale, e tanto più sia difficile a conservare nell'espressioni, quanto la lingua è più ricca, è verità tanto evidente che fu la prima di cui io m'accorsi quando cominciai a riflettere seriamente sulla letteratura: e dopo questo facilmente vidi che il mezzo più spedito e sicuro di ottenere questa proprietà era il trasportare d'una in altra lingua i buoni scrittori. ${ }^{23}$

A tradução foi usada por Leopardi para aprender outras línguas, como o grego e o latim, mas também para experimentar novas formas literárias e para se formar como escritor. Assim, em uma carta de 29 de dezembro de 1817, endereçada ao escritor e amigo Pietro Giordani, Leopardi diz:

(...) E in oltre mi pare d'essermi accorto che il tradurre così per esercizio vada veramente fatto innanzi al comporre, e o bisogni o giovi assai per divenire insigne scrittore, ma che per divenire insigne traduttore convenga prima aver composto ed essere bravo scrittore, e che in somma una traduzione perfetta sia opera più tosto da vecchio che da giovane. ${ }^{24}$

Nessa carta, Leopardi expõe uma concepção original, defendendo a prática da tradução para o escritor iniciante. Segundo ele, é traduzindo que se aprende a compor com estilo. Convém frisar que quando o poeta de "L'Infinito" fala que é traduzindo que se aprende a escrever, ele se refere à tradução de grandes autores clássicos gregos e latinos, como Homero, Virgílio e Horácio. Para Leopardi, quando um tradutor é um escritor que escreve bem, a probabilidade de uma boa tradução é bastante alta, pois a tradução de qualidade é obra do escritor maduro.

\footnotetext{
${ }^{21}$ DE SANCTIS. Opere, p. 1140.

${ }^{22}$ LEOPARDI. Epistolario, p. 48.

${ }^{23}$ LEOPARDI. Epistolario, p. 94.

${ }^{24}$ LEOPARDI. Epistolario, p. 172.
} 
Como se sabe, essa postura de Leopardi será a mesma adotada, mais tarde, por Pound e outros escritores, para quem somente um bom escritor-poeta pode traduzir outro bom escritor-poeta. No prefácio da tradução do segundo livro da Eneida, Leopardi é mais enfático e afirma que "(...) senza esser poeta non si può tradurre un vero poeta". ${ }^{25}$

As reflexões teóricas de Leopardi sobre tradução literária nascem de sua experiência como tradutor de diferentes literaturas, mas principalmente da grega e da latina. Essa relação com outras literaturas através da tradução faz com que não apenas aprenda a língua, mas incorpore estilos e formas.

Assim, no início da carreira, Leopardi compõe obras de caráter filológico e ensaios, até evoluir para as traduções de Mosco, Homero, Virgílio, e a se descobrir, então, como poeta e prosador. Essa trajetória pode ser comparada à de muitos escritores, como a de Jorge Luis Borges, que também foi escritor precoce e iniciou a sua vida literária fazendo traduções, para depois se tornar poeta e prosador. E em uma carta de 21 de março de 1817 ao escritor e amigo Pietro Giordani, diz categoricamente: "Se io vivrò, vivrò alle Lettere, perchè ad altro non voglio nè potrei vivere". ${ }^{26}$

Como se percebe, Leopardi guarda uma relação íntima com a tradução em seus mais variados aspectos. Sua formação literária aconteceu graças ao seu contato estreito com o texto traduzido, seguindo assim a tradição de muitos grandes autores em que, por exemplo, a tradução de clássicos gregos e latinos servia não apenas para incorporar novas formas literárias, mas também para renovar a sua própria.

Não por acaso, Sainte-Beuve, o principal divulgador de Leopardi na França, ${ }^{27} \mathrm{em}$ Portrait de Leopardi, descreve o escritor italiano como um homem de pensamento superior e o classifica como o último dos antigos. ${ }^{28} \mathrm{E}$ acrescenta: "Il nous semble qui si, par ses audaces et ses rajeunissements de langage, par son culte de la forme retrouvée, Leopardi appartient à l'école des novateurs, il était du moins le classique par excellence entre les romantiques." 29

Assim, Leopardi pode ser considerado o mais moderno entre os grandes autores de tradição clássica e isso se deve à sua capacidade, conjugada pelos elementos "fantasia, raciocínio e dor", de "canibalisticamente" assimilar, através da tradução, o estilo dos melhores escritores gregos e latinos.

\section{A}

\footnotetext{
${ }^{25}$ LEOPARDI. Epistolario, p. 554.

${ }^{26}$ LEOPARDI. Epistolario, p. 72.

${ }^{27}$ Dionisotti afirma que Sainte-Beuve é o responsável pela virada decisiva na fortuna crítica de Leopardi "sottraendolo alla meschinità del culto municipale e partigiano, che gli era toccato in sorte in Italia, e isolandolo, secondo la verità storica, come autore vissuto e vivente in Europa". In: DIONISOTTI. Appunti sui moderni: Foscolo, Leopardi, Manzoni e altri, p. 220.

${ }^{28}$ SAINTE-BEUVE. Portrait de Leopardi, p. 44.

${ }^{29}$ SAINTE-BEUVE. Portrait de Leopardi, p. 37.
} 


\section{A B STRACT}

This article analyzes the relation between Italian writer Giacomo Leopardi (1798-1837) and authors from Classical Antiquity, as well as how his translating Greek and Roman authors contributed to his intellectual development, and helped him become one of the most important writers in nineteenth century Italy.

\section{KEYWORDS}

Leopardi, Translation, Classical Antiquity Literature

\section{REFERÊNCIAS}

BOLLATI, Giulio. Giacomo Leopardi e la letteratura italiana. Torino: Bollati Boringhieri, 1998. 158 p.

BOSI, Alfredo. A natureza, os antigos: Leopardi Tradutor. In: LUCCHESI, Marco (Org.). Giacomo Leopardi Poesia e Prosa. Rio de Janeiro: Nova Aguilar, 1996. p. 158-173.

DAMIANI, Rolando. All'apparir del vero - vita di Giacomo Leopardi. Milano: Mondadori, 2002. 529 p.

DE SANCTIS, Francesco. Studio su Giacomo Leopardi. Recanati: Osanna Venosa, 2001. $391 \mathrm{p}$.

DE SANCTIS, Francesco. Opere. Milano/Napoli: Riccardo Ricciardi Editore, 1986. 1.316 p. DIONISOTTI, Carlo. Appunti sui moderni: Foscolo, Leopardi, Manzoni e altri. Bolonha: Mulino, 1988. 402 p.

DOTTI, Ugo. Storia della letteratura italiana. Roma: Carocci, 2007. 596 p.

LEOPARDI, Giacomo. Epistolario. A cura di Franco Brioschi e Patrizia Landi. Torino: Bollati Boringhieri, 1998. 2 v. 2.540 p.

LEOPARDI, Giacomo. Zibaldone di Pensieri. Disponível em: < http://www.classicitaliani.it/ leopardi/Zibaldone/Leopardi_Zibaldone_1677_1914.htm >. Acesso em: 19 fev. 2009.

SAINTE-BEUVE. Portrait de Leopardi. Paris: Allia, 1994. 70 p.

POLATO, Lorenzo. Il sogno di um'ombra: Leopardi e la verità delle illusioni. Venezia: Marsilio, 2007. 126 p.

PRETE, Antonio. Il pensiero poetante. Saggio su Leopardi. Milano: Universale Economica Feltrinelli, 2006. 203 p.

PRETE, Antonio. Finitudine e Infinito su Leopardi. Milano: Feltrinelli, 1998. 174 p.

RANIERI, Antonio. Sette anni di sodalizio com Giacomo Leopardi. Milano: SE, 2005. 128 p.

RANIERI, Antonio. Notizia intorno alla vitta e agli scritti di Giacomo Leopardi. In: . Sette anni di sodalizio com Giacomo Leopardi. Milano: SE, 2005. 128 p. 